\title{
THE STRUCTURE OF THE FIXED POINT SET OF QUADRATIC OPERATORS ON THE SIMPLEX
}

\author{
MANSOOR SABUROV*, ${ }^{* *}$ AND NUR ATIKAH YUSOF*** \\ * Department of Computational \& Theoretical Sciences \\ International Islamic University Malaysia \\ 25200 Kuantan, Pahang, Malaysia \\ E-mail: msaburov@gmail.com \\ ** Department of Mathematics and Statistics, \\ College of Engineering and Technology, \\ American University of Middle East, 250St., Egaila, Kuwait \\ ***Department of Computational \& Theoretical Sciences \\ International Islamic University Malaysia \\ 25200 Kuantan, Pahang, Malaysia \\ E-mail: y.nuratikah@gmail.com
}

\begin{abstract}
We know that any linear operator associated with a positive square stochastic matrix has a unique fixed point in the simplex. However, in general, the similar result for a quadratic operator acting on the simplex does not hold true. Namely, there is a quadratic operator associated with a positive cubic stochastic matrix which has more than one fixed point in the simplex. The first attempt to give an example for such kind of quadratic operators was done by A.A. Krapivin and Yu.I. Lyubich. However, we showed that their examples are wrong. Therefore, in this paper, we decided to give a correct example for a quadratic operator with positive coefficients having three fixed points in the simplex. Moreover, we also describe the number of fixed points of the quadratic operator associated with a positive cubic stochastic matrix.
\end{abstract}

Key Words and Phrases: Cubic stochastic matrix, quadratic stochastic operator, fixed point.

2010 Mathematics Subject Classification: 47H10, 37C25, 58C30.

Acknowledgment. This work was partially supported by the MOHE grant FRGS14141-0382. The first author (M.S.) was supported by the Junior Associate scheme of the Abdus Salam International Centre for Theoretical Physics (ICTP), Trieste, Italy.

\section{REFERENCES}

[1] S. Bernstein, Solution of a mathematical problem connected with the theory of heredity, Annals of Math. Statistics, 13(1942), 53-61.

[2] N. Ganikhodjaev, R. Ganikhodjaev, U. Jamilov, Quadratic stochastic operators and zero-sum game dynamics, Ergodic Th. and Dynamical Systems, 35(2015), no. 5, 1443-1473.

[3] N. Ganikhodjaev, M. Saburov, U. Jamilov, Mendelian and Non-Mendelian quadratic operators, App. Math. Info. Sci., 7(2013), no. 5, 1721-1729.

[4] N. Ganikhodjaev, M. Saburov, A.M. Nawi, Mutation and chaos in nonlinear models of heredity, The Scientific World J., 2014(2014), 1-11. 
[5] R. Ganikhodzhaev, F. Mukhamedov, U. Rozikov, Quadratic stochastic operators and processes: Results and Open Problems, Inf. Dim. Anal. Quan. Prob. Rel. Top., 14(2011), no. 2, 279-335.

[6] R. Ganikhodjaev, M. Saburov, Kh. Saburov, Schur monotone decreasing sequences, AIP Conference Proceedings, 1557(2013), 108-111.

[7] H. Kesten, Quadratic transformations: A model for population growth I, Adv. App. Prob., 2(1970), 1-82.

[8] A.A. Krapivin, Fixed points of quadratic operators with positive coefficients, Theory of Functions, Functional Anal. Appl., 25(1975), 62-67.

[9] C.-K. Li, S. Zhang, Stationary probability vectors of higher-order Markov chains, Linear Algebra App., 473(2015), 114-125.

[10] Yu.I. Lyubich, Mathematical Structures in Population Genetics, Springer-Verlag, 1992.

[11] F. Mukhamedov, M. Saburov, On homotopy of volterrian quadratic stochastic operator, App. Math. Info. Sci., 4(2010), 47-62.

[12] F. Mukhamedov, M. Saburov, On dynamics of Lotka-Volterra type operators, Bull. Malay. Math. Sci. Soc, 37(2014), 59-64.

[13] F. Mukhamedov, M. Saburov, I. Qaralleh, On $\xi^{s}$-quadratic stochastic operators on twodimensional simplex and their behavior, Abstract Applied Anal., 2013(2013), 942038.

[14] M. Saburov, Some strange properties of quadratic stochastic Volterra operators, World Applied Science J., 21(2013), 94-97.

[15] M. Saburov, A class of nonergodic Lotka-Volterra operators, Math. Notes, 97(2015), no. 5, 759-763.

[16] M. Saburov, On divergence of any order Cesaro mean of Lotka-Volterra operators, Ann. Funct. Anal., 6(2015), no. 4, 247-254.

[17] M. Saburov, Kh. Saburov, Mathematical models of nonlinear uniform consensus, Science Asia, 40(2014), no. 4, 306-312.

[18] M. Saburov, Kh. Saburov, Reaching a nonlinear consensus: Polynomial stochastic operators, Inter. J. Cont. Auto. Systems, 12(2014), no. 6, 1276-1282.

19] B. Sturmfels, Solving Systems of Polynomial Equations, Texas, 2002

[20] S. Ulam, A Collection of Mathematical Problems, New-York, London 1960.

Received: June 30, 2015; Accepted: December 11, 2015. 